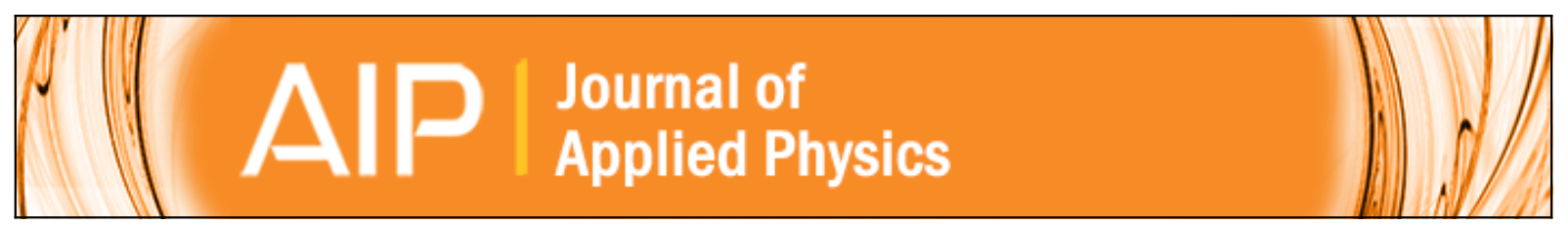

\title{
Overcoming the adverse effects of substrate on the waveguiding properties of plasmonic nanoparticle chains
}

Ilia L. Rasskazov, Sergei V. Karpov, George Y. Panasyuk, and Vadim A. Markel

Citation: Journal of Applied Physics 119, 043101 (2016); doi: 10.1063/1.4940415

View online: http://dx.doi.org/10.1063/1.4940415

View Table of Contents: http://scitation.aip.org/content/aip/journal/jap/119/4?ver=pdfcov

Published by the AIP Publishing

\section{Articles you may be interested in}

Numerical investigation of optical Tamm states in two-dimensional hybrid plasmonic-photonic crystal nanobeams J. Appl. Phys. 116, 043106 (2014); 10.1063/1.4891222

Plasmonic modes and extinction properties of a random nanocomposite cylinder

Phys. Plasmas 21, 042112 (2014); 10.1063/1.4871495

Hybrid nano ridge plasmonic polaritons waveguides

Appl. Phys. Lett. 103, 131107 (2013); 10.1063/1.4823546

Plasmonic waveguide as an efficient transducer for high-density data storage

Appl. Phys. Lett. 95, 171112 (2009); 10.1063/1.3257701

Surface plasmon dielectric waveguides

Appl. Phys. Lett. 87, 241106 (2005); 10.1063/1.2142096

\section{Đ SHIMADZU Powerful, Multi-functional UV-Vis-NIR and Excellence in science FTIR Spectrophotometers}

Providing the utmost in sensitivity, accuracy and resolution for applications in materials characterization and nano research

- Photovoltaics - Ceramics

- Polymers

- Thin films

- Paints

- DNA film structures

- Coatings

- Packaging materials
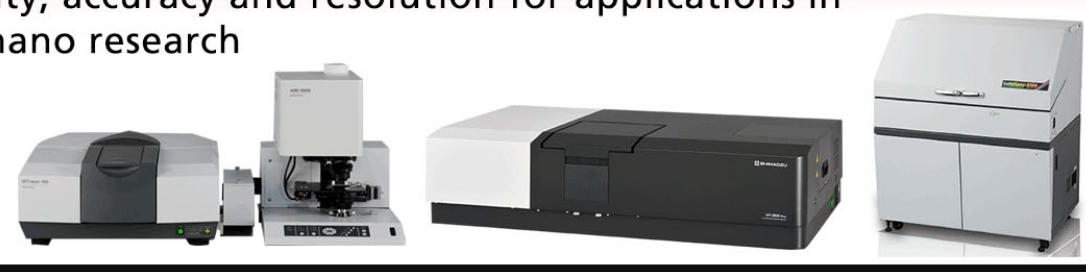


\title{
Overcoming the adverse effects of substrate on the waveguiding properties of plasmonic nanoparticle chains
}

\author{
Ilia L. Rasskazov, ${ }^{1}$ Sergei V. Karpov, ${ }^{1,2,3}$ George Y. Panasyuk, ${ }^{4}$ and Vadim A. Markel ${ }^{5, a)}$ \\ ${ }^{1}$ Siberian Federal University, 660041 Krasnoyarsk, Russia \\ ${ }^{2}$ L. V. Kirensky Institute of Physics, 660036 Krasnoyarsk, Russia \\ ${ }^{3}$ Siberian State Aerospace University, 660014 Krasnoyarsk, Russia \\ ${ }^{4}$ Aerospace Systems Directorate, Air Force Research Laboratory, Wright-Patterson Air Force Base, \\ Ohio 45433, USA \\ ${ }^{5}$ Aix-Marseille Université, CNRS, Centrale Marseille, Institut Fresnel UMR 7249, 13013 Marseille, France
}

(Received 15 October 2015; accepted 10 January 2016; published online 22 January 2016)

We have studied numerically the propagation of surface plasmon polaritons (SPPs) in linear periodic chains of plasmonic nanoparticles of different shapes. The chains are deposited on top of a thick dielectric substrate. While in many commonly considered cases the substrate tends to suppress the SPP propagation, we have found that this adverse effect is practically absent in the case when the nanoparticles have the shape of oblate spheroids with sufficiently small aspect ratio (e.g., nanodisks) whose axes of symmetry coincide with the axis of the chain. (C) 2016 AIP Publishing LLC.

[http://dx.doi.org/10.1063/1.4940415]

\section{INTRODUCTION}

Linear or curved chains of metallic nanoparticles (plasmonic chains) and two-dimensional periodic arrangements of nanoparticles on planar substrates have attracted significant recent attention, mainly, in conjunction with spectroscopic and sensing applications. ${ }^{1-10}$ The useful feature of the physical objects mentioned above is their capacity to support very narrow non-Lorentzian resonances ${ }^{11}$ that are not broadened by Ohmic losses but very sensitive to the chemical environment. These spectral lines have been observed experimentally. Waveguiding applications of plasmonic chains have also been considered in the literature. ${ }^{12-19}$ The major problems one faces in the waveguiding applications are (i) engineering of useful dispersion relations (ii) coping with losses and (iii) coping with parasitic electromagnetic coupling with the environment, in particular, with the substrate. In our previous work, we have used the additional degrees of freedom that are inherent in nonspherical particles such as oblate and prolate spheroids to solve some of these problems. ${ }^{20-22}$ We have found that, generally, chains of oblate spheroids (nanodisks) whose short axes are aligned with the axis of the chain can overcome many of the existing difficulties. In particular, such waveguides are characterized by high field localization, dispersion curves of sufficient slope to form well-defined wave packets and by efficient propagation even in the presence of Ohmic losses. However, so far we have considered free chains in vacuum. This is not realistic because, in practice, the chains must be deposited on a substrate. In this paper, we study the effects of a dielectric substrate such as glass on the propagation of surface plasmon polaritons (SPPs) in chains composed of spheroidal particles of various shapes. Again, we find that the chains composed

\footnotetext{
${ }^{a)}$ On leave from the Department of Radiology, University of Pennsylvania, Philadelphia, Pennsylvania 19104, USA. Electronic mail: vmarkel@mail. med.upenn.edu
}

of nanodisks do not experience the adverse effects of the substrate.

SPPs in plasmonic chains are currently a mature research subject. Propagation and decay of SPPs in infinite or semi-infinite linear, perfectly periodic chains are well understood at present. ${ }^{21,23,24}$ Although a perfectly periodic chain is an idealization, it was shown that weak disorder $^{23,25,26}$ does not have a dramatic effect on long-range SPP propagation. Dispersion relations and transient processes (wave packet transmission) in plasmonic chains have also been studied in detail. ${ }^{19,20,27-29}$ In particular, it was found that chains of spherical particles cannot support propagation of well-formed wave packets due to the flatness of the corresponding dispersion curves. This, in particular, has motivated our interest in chains of non-spherical particles such as prolate or oblate spheroids, ${ }^{20,21}$ which can serve as broadband SPP waveguides. Moreover, curved chains of spheroids exhibit unique properties such as high anisotropy and polarization sensitivity. ${ }^{30}$

In the majority of theoretical works on the subject, plasmonic chains were assumed to be in vacuum or embedded in a spatially uniform host medium. However, the practical use of plasmonic chains requires their deposition on a flat substrate. Therefore, the effect of the substrate on the waveguiding properties of plasmonic chains is of practical importance. This question was addressed recently in a number of publications. In Refs. 4, 18, and 31, the optical properties of linear and curved chains of spherical nanoparticles located near a metal $(\mathrm{Ag}, \mathrm{Au})$ substrate were investigated. In Ref. 32, coupling of the surface modes of a dielectric slab and a plasmonic chain were considered from the theoretical point of view (here, one important consideration is the momentum conservation law, which can allow or forbid coupling of different modes depending on the respective dispersion relations). Generally, it can be concluded from these works that substrates play a detrimental role by suppressing propagation 
of SPPs or coupling them to other surface modes, which can be an unintended effect.

However, none of the above works considered nonspherical particles. We therefore have computed the transmission characteristics of plasmonic chains composed of spheres, prolate and oblate spheroids on a dielectric substrate numerically. We emphasize that the present paper is primarily computational. Thus, we do not utilize the theoretical arguments such as those employed in Ref. 32, although such considerations can be very illuminating. Our goal here is simply to investigate the transmission properties of various chains, which is achievable numerically.

Below, in Sec. II, we summarize the theoretical model that we use. As many other authors, we work in the dipole approximation. The major problem in this case is the computation of the reflected part of the electromagnetic Green's tensor (due to the presence of the substrate) between all possible pairs of particles and between each particle and itself. The later term describes the substrate-mediated self-interaction of a particle, ${ }^{33}$ which we take fully into account. Sometimes, the problem mentioned above is tackled by utilizing the approximate electrostatic method of images. ${ }^{34}$ However, this approximation is inaccurate for particle pairs with large lateral separations-a consideration that is important for the theoretical description of long-range SPPs. Therefore, we have utilized a purely numerical approach to compute the Sommerfeld integral by discretization. In Sec. III, we present our numerical results. Finally, in Sec. IV, we give a summary of the obtained results.

\section{MODEL}

We work in the frequency domain (frequency $\omega$, free space wave number $k=\omega / c)$, and the factor $\exp (-i \omega t)$ is suppressed in all expressions. We consider linear periodic chains of metallic nanoparticles (spheres, prolate and oblate spheroids) of the permittivity $\epsilon_{m}$ whose centers are located at the height $\mathcal{H}$ above a planar dielectric substrate with the permittivity $\epsilon_{d}$. We assume that there is no other host material or medium above the substrate. The center-to-center separation of neighboring spheroids is denoted by $h$, and the longer and shorter semiaxes of each spheroid are denoted by $a$ and $b$. In the case of spheres, $a=b$. A given waveguide contains $N$ identical spheroids arranged in a perfectly periodic chain. In this paper, we do not consider disordered or curved chains as were done in some of our previous publications. ${ }^{22,23}$

We arrange the spheroids so that the shorter spheroid axes are always parallel to the axis of the chain. Graphical illustrations of this arrangement are given below. This geometry is characterized by a more efficient electromagnetic coupling of the neighboring spheroids and, therefore, by a more efficient SPP propagation through the waveguide. ${ }^{21,22,35}$

More specifically, in the case of oblate spheroids, the two longer spheroid semiaxes are oriented orthogonally to the chain. In vacuum, this waveguide would have been cylindrically symmetric. However, in the presence of a substrate, this symmetry is broken and the two transversely polarized SPPs propagating in this chain are no longer equivalent. Moreover, the SPP that is polarized transversely to both the chain and the substrate is coupled to the SPP that is polarized parallel to both the chain and the substrate. This coupling is mediated by the substrate and its physical consequence is polarization conversion of SPPs upon propagation. Thus, we see that the transversely and longitudinally polarized SPPs are no longer decoupled in the presence of the substrate even in perfectly linear chains. However, note that the SPP that is polarized transversely to the chain but parallel to the substrate is decoupled from other modes and does not experience polarization conversion.

In the case of prolate spheroids, the chain does not possess cylindrical symmetry even in the absence of the substrate. If the substrate is present, there are two possible cases to consider: the longer spheroid axes can be either parallel or orthogonal to the substrate. In each arrangement, there are two distinct transversely polarized SPPs, so that the total number of different SPPs with transverse polarization that we can consider is four. As in the case of oblate spheroids, the SPPs that are polarized transversely to both the chain and the substrate are coupled to the longitudinally polarized SPPs.

We have used the following physical parameters in the simulations. The shorter semiaxes of all spheroids (in the special case of spheres, the radiuses) are set to be $b=8 \mathrm{~nm}$, and the center-to-center separation of the neighboring nanoparticles is $h=3 b=24 \mathrm{~nm}$. Therefore, the surface-to-surface separation of neighboring particles is in all cases equal to $b$. The height above the substrate varied in the simulation but, for the most part, we compare the case when the particles are touching the substrate with the case of free chains (obtainable in the limit $\mathcal{H} \rightarrow \infty$ ).

The permittivity of the particles is assumed to obey the Drude formula

$$
\epsilon_{m}=\epsilon_{\infty}-\frac{\omega_{p}^{2}}{\omega(\omega+i \gamma)},
$$

where $\epsilon_{\infty}=5$ and $\omega_{p} / \gamma=526.3$, which approximately corresponds to silver for the wavelengths larger than $\approx 400 \mathrm{~nm}$. The permittivity of the substrate is taken to be frequency independent but complex, i.e., $\epsilon_{d}=2.5+0.01 i$, which corresponds, approximately, to glass. Thus, we disregard frequency dispersion in the substrate but take into account dispersion in the metal.

Modeling is performed in the dipole approximation. Each nanoparticle is characterized by the location of its center $\mathbf{r}_{n}$ and the dipole moment $\mathbf{d}_{n}$, where $n=1,2, \ldots, N$ and $N$ is the total number of nanoparticles in a finite chain, and we assume that the point dipole $\mathbf{d}_{n}$ is located at $\mathbf{r}_{n}$. The coupleddipole equations in the presence of the substrate are of the form

$$
\mathbf{d}_{n}=\alpha\left[\mathbf{E}_{n}+\sum_{m=1}^{N}\left(G_{n m}^{F}+G_{n m}^{R}\right) \mathbf{d}_{m}\right] .
$$

Here, $\mathbf{E}_{n}$ is the electric field produced by all external sources at the center of $n$-th nanoparticle, $\alpha$ is the polarizability tensor (same for all nanoparticles for the geometry considered), $G_{n m}^{F}$ is the regular part of the free-space Green's tensor, and $G_{n m}^{R}$ is the contribution to the Green's tensor due to the substrate (the "reflected" part). Note that, by definition, $G_{n n}^{F}=0$ 
but $G_{n n}^{R}$ is generally different from zero; summation in (2) runs over all indexes including the index $m=n$. The latter term in the summation accounts for the substrate-mediated self-interaction of each dipole. In this approach, the polarizability of the particles $\alpha$ is defined with respect to the general external field, which includes the field reflected by the substrate. We can refer to the quantity $\alpha$ as to the polarizability of a "free" particle. Mathematical expressions for $\alpha$ that we use in this paper are given in Ref. 22 and take into account second-order ${ }^{36}$ and third-order ${ }^{37}$ (in the wave number $k$ ) corrections to the quasi-static polarizability tensor of a spheroid.

Note that there exists an alternative formulation of the coupled-dipole equation in which the summation in the right-hand size of (2) runs over the indexes $m \neq n$ and the effective ("renormalized") polarizability

$$
\alpha_{n}^{\text {eff }}=\left(I-\alpha G_{n n}^{R}\right)^{-1} \alpha
$$

is introduced. ${ }^{33}$ In the above equation, $I$ is the identity tensor, and the renormalized polarizability depends on the distance to the substrate. The quantity $\alpha_{n}^{\text {eff }}$ can be understood as the linear response coefficient with respect to all external fields except for the fields reflected by the substrate. The mathematical equivalence of these two approaches can be established by moving the diagonal term from the summation of Equation (2) to the left-hand side. We note that the formulation (2) is more convenient in numerical simulations involving identical but arbitrarily located particles because it contains a single spectral parameter $\alpha$ that is independent of $n$. However, if the particles are physically different, this advantage is lost.

The dipole approximation is widely used in the literature and is valid for nanoparticles that are separated by sufficiently large distance. The particle separation at which the dipole approximation breaks depends on the polarization and, generally, it is rather small for transverse (with respect to the chain) polarizations. The alternative to the dipole approximation is the use of rigorous numerical methods such as finite difference or finite elements methods. Unfortunately, application of these methods to long chains (e.g., longer than $\sim 10$ nanoparticles) is technically problematic and subject to uncertainties. For example, the exact shape of the particles is rarely known or described by regular geometrical shapes. Also, solution of the electromagnetic boundary value problem in nanoparticles is affected by the quantum finite-size effects that are difficult to incorporate into the FD (finite difference) or FEM (finite element method) schemes. In the dipole approximation that we use, the exact shape of the particles is not overly important and the finite size effects can be, in principle, incorporated into a single parameter, the particle polarizability. We therefore believe that the dipole approximation captures the essential physics of plasmonics chains reasonably well and serves as a useful mathematical model. We also emphasize that spheroids are used below only as examples; the exact spheroidal shape is not important. What is important is the depolarization factors of the nanoparticles that ultimately determine their polarizabilities. For example, we can use nanodisks (truncated cylinders) instead of oblate spheroids, the only requirement being that the two shapes have a dominating dipole resonance at approximately the same frequency.

Expression for $G_{n m}^{F}$ is simple and well-known. The tensor $G_{n m}^{R}$ for the half-space above the substrate can be written in the form of a Fourier integral. ${ }^{38,39}$ Mathematical expressions for $G_{n m}^{F}$ and $G_{n m}^{R}$ are also given in Ref. 40. To use these expressions directly, we should apply the correspondence rules $G_{n m}^{F} \leftarrow \mathcal{G}^{F}\left(\mathbf{r}_{n}-\mathbf{r}_{m}\right)$, where $\mathcal{G}^{F}(\mathbf{r})$ is given in Eq. 8 of Ref. 40 with $k_{1} \rightarrow k=\omega / c$ and $G_{n m}^{R} \leftarrow G^{R}\left(\mathbf{r}_{n}, \mathbf{r}_{m}\right)$, where the latter quantity is given by Eq. 10 in Ref. 40. We should keep in mind that $G_{n n}^{F}=0$ by definition, and we should not attempt to use the mathematical expression for $\mathcal{G}^{F}(\mathbf{r})$ to compute this quantity (which is obviously impossible).

In some special cases, the electrostatic method of reflections or its analytical extensions ${ }^{40}$ can be used to compute $G_{n m}^{R}$ approximately. However, for the problem at hand, the electrostatic reflection formulas as well as its analytical extensions are grossly inaccurate, particularly, for describing the interaction of particles that are separated by a lateral distance that is significantly larger than the free space wavelength. In this work, we use a purely numerical approach to computing $G_{n m}^{R}$. Specifically, we compute the Fourier integral numerically by discretization.

Solution to (2) can be written in the form

$$
\mathbf{d}_{n}=\sum_{m} \mathcal{D}_{n m} \mathbf{E}_{m},
$$

where $\mathcal{D}_{n m}$ is the Green's function for the chain. Here, each element $\mathcal{D}_{n m}$ is a $3 \times 3$ tensor. For a linear periodic chain in vacuum, all tensors $\mathcal{D}_{n m}$ are diagonal in a Cartesian reference frame that has one of its axes parallel to the axis of the chain. In curved chains, this is no longer so. ${ }^{22}$ In a linear chain above the substrate, diagonality is also lost. For example, in the reference frame shown in Fig. 1 below, $\left(\mathcal{D}_{n m}\right)_{z y}$ $=\left(\mathcal{D}_{n m}\right)_{y z} \neq 0$ while other non-diagonal elements of $\mathcal{D}_{n m}$ are zero.

If the first particle is illuminated by an external field of amplitude $\mathbf{E}_{1}=\mathbf{A}$ and all other amplitudes $\mathbf{E}_{m}$ with $m \neq 1$ are zero, then the solution to (2) takes the form $\mathbf{d}_{n}=\mathcal{D}_{n 1} \mathbf{A}$. To quantify the transmission properties of a chain, we will use the normalized Green's functions ${ }^{22,23}$

$$
\mathcal{F}_{n}=\frac{\left|\mathcal{D}_{n 1} \mathbf{A}\right|}{\left|\mathcal{D}_{11} \mathbf{A}\right|},
$$

where the length of an arbitrary complex vector $\mathbf{x}$ is $|\mathbf{x}|=\sqrt{\mathbf{x}^{*} \cdot \mathbf{x}}$. For a chain consisting of $N$ particles, we

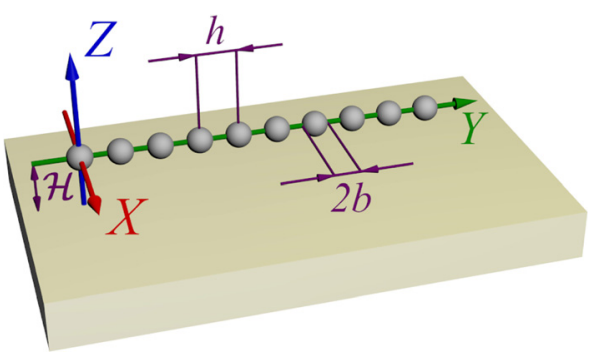

FIG. 1. Schematic view of a chain composed of spherical particles. 
will refer to the positive scalar quantity $\mathcal{F}_{N}$ as to the transmission of the chain. According to this terminology, the dependence of $\mathcal{F}_{N}(\omega)$ on the electromagnetic frequency $\omega$ (for a stationary monochromatic excitation) is the transmission spectrum.

\section{NUMERICAL RESULTS}

\section{A. Spherical particles}

We start with the case of chains made from spherical particles (see Fig. 1 for an illustration). The transmission spectra $\mathcal{F}_{N}(\omega)$ for chains consisting of $N=1001$ nanospheres and for various heights of the chain above the substrate (as well as in the absence of the substrate) are shown in Fig. 2. In this and other figures below, we classify the results by the polarization of the external field amplitude, $\mathbf{A}$, which is assumed to be strictly linear. In some cases, this polarization coincides with the polarization of the excited SPP. In other cases, the SPP undergoes polarization conversion as will be shown below. In labeling the figures, we will always refer to the polarization of $\mathbf{A}$, which can also be thought of as the "initial" polarization of the SPP.

It can be seen from Fig. 2 that the SPP transmission is strongly suppressed by the substrate for both transverse

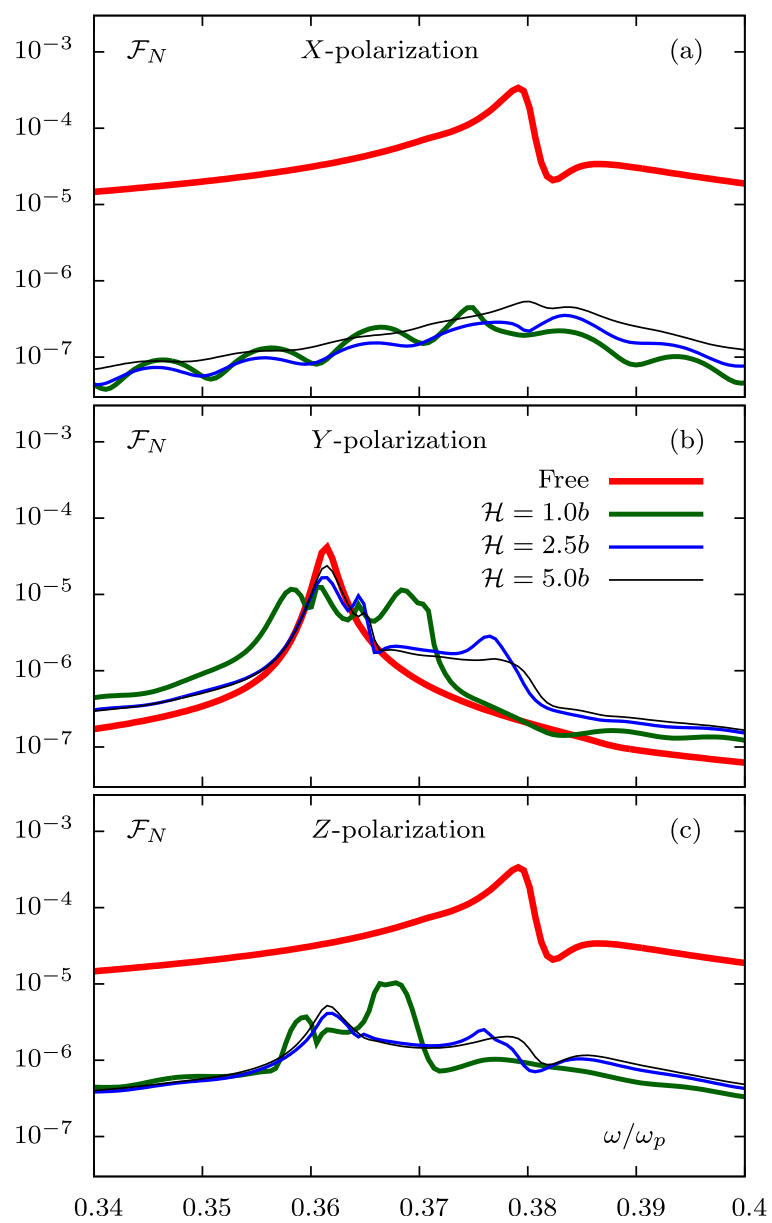

FIG. 2. Transmission spectra for a chain of $N=1001$ spherical particles for different values of the height above the substrate $\mathcal{H}(\mathcal{H}=b$ corresponds to touching) and for different directions of the external field $\mathbf{A}$. The thick (red) lines give the transmission spectra without the substrate. polarizations (along $X$ and $Z$ axes). The strongest suppression occurs for the $X$-polarization (in plane of the substrate). In this case, not only the transmission is suppressed but also its spectrum does not exhibit the resonant behavior, which is characteristic of the transmission spectra of chains in vacuum. However, in the case of longitudinal polarization (along the $Y$-axis), the effect of the substrate is much less pronounced, and no suppression of transmission is observed. At some frequencies, the transmission is in fact enhanced by the substrate.

If we excite the chain by $Y$ or $Z$-polarized field, the resulting SPP undergoes polarization conversion. This is so because the SPPs that are polarized orthogonally to both the chain and the substrate are electromagnetically coupled to the longitudinally polarized SPPs. Mathematically, the coupling takes place due to the nonzero elements $\left(G_{n m}^{R}\right)_{y z}$ $=\left(G_{n m}^{R}\right)_{z y}$. All other off-diagonal elements of the tensors $G_{n m}^{R}$ are zero. Therefore, polarization conversion does not occur for the $X$-polarization.

We quantify the polarization conversion effect by the ratio $\delta_{n}$ defined as

$$
\delta_{n}= \begin{cases}\left|d_{n z}\right| /\left|d_{n y}\right| & \text { for } \mathbf{A} \text { along } Y \\ \left|d_{n y}\right| /\left|d_{n z}\right| & \text { for } \mathbf{A} \text { along } Z\end{cases}
$$

In other words, $\delta_{n}$ quantifies the conversion of the initial polarization created by the external field into an orthogonal polarization due to the interaction with the substrate. Note that in the definition (6), we disregard the phase of the dipole amplitude ratios, and therefore we do not distinguish between an elliptical and linear (but rotated) polarizations. The results for $\delta_{n}$ are shown in Fig. 3. It can be seen that $\delta_{n}$

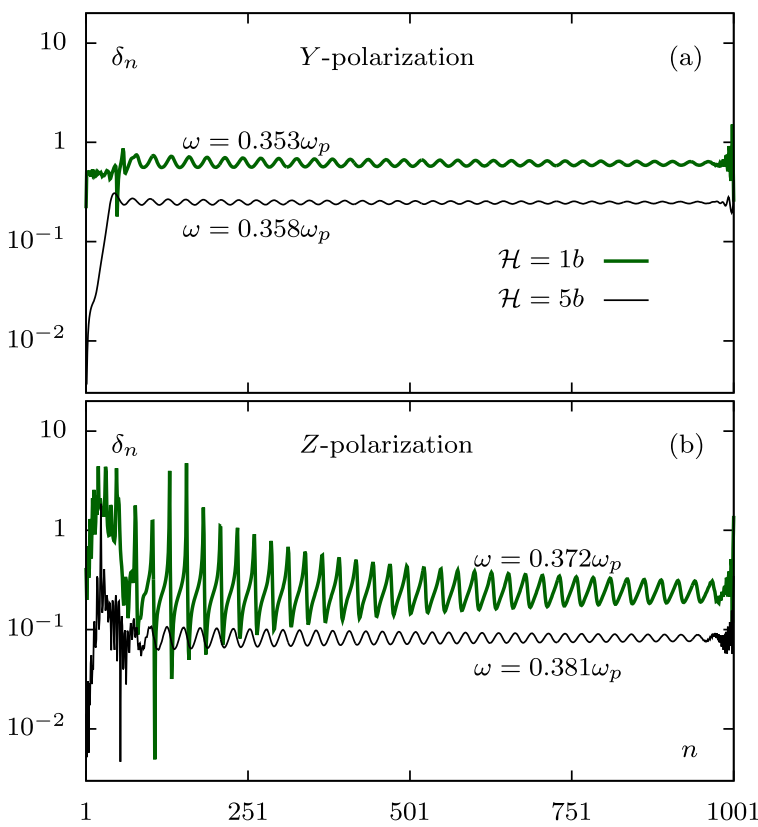

FIG. 3. Depolarization ratio $\delta_{n}$ (6) for a chain of $N=1001$ spherical particles for different polarizations of the external field $\mathbf{A}$ and for different heights $\mathcal{H}$ above the substrate ( $\mathcal{H}=b$ corresponds to touching). In each case, the SPPs are excited at the frequencies (as labeled) that correspond to the maximum transmission. These frequencies were determined from the data of Fig. 2. 
approaches unity when the chain touches the substrate $(\mathcal{H}=b)$ indicating that the effects of polarization conversion are in this case very strong. When $\mathcal{H}=5 b, \delta_{n}$ does not deviate too much from zero. Theoretically, $\delta_{n}=0$ in the absence of the substrate (these results are trivial and the corresponding data are not shown).

\section{B. Prolate spheroids}

Now we turn to the case of prolate spheroids. Note that we consider the same geometrical chain parameters as were used by us previously. ${ }^{21}$ In particular, the longer semiaxes of the spheroids are always orthogonal to the chain. However, when the chain is placed close to a substrate, there are additional options for the chain arrangement, as shown in Fig. 4. We will consider here both possible cases in which the longer semiaxes of the spheroids are (a) parallel and (b) orthogonal to the substrate. Also, the transmission spectra of chains consisting of spheroids with the aspect ratios $\xi=b / a>0.6$ are fairly similar to those in the chains of spherical particles. Therefore, we will consider spheroids with a significantly smaller aspect ratio, namely, $\xi=b / a=0.2$.

Transmission spectra of the chains shown in Fig. 4(a) are given in Fig. 5. For the $X$-polarization, transmission is strongly suppressed by the substrate at all frequencies. However, for $Y$ and $Z$ polarizations, there exist resonance frequencies at which the transmission is not much worse than the best (maximum) transmission of a free chain. This indicates that the frequency can be tuned so that the substrate does not play a very detrimental role.

The results for prolate spheroid having their axes of symmetry oriented perpendicular to the plane of the substrate (as in Fig. 4(b)) are shown in Fig. 6. Here, we also compare a chain that is touching the substrate and a free chain. As before, transmission is strongly suppressed in $X$-polarization for all frequencies. However, the results are much more promising in $Y$ and $Z$ polarizations. Here, the substrate does not play such a detrimental role.

The stronger transmission in the chains shown in Fig. 4(b) compared to those shown in Fig. 4(a) can be

(a)
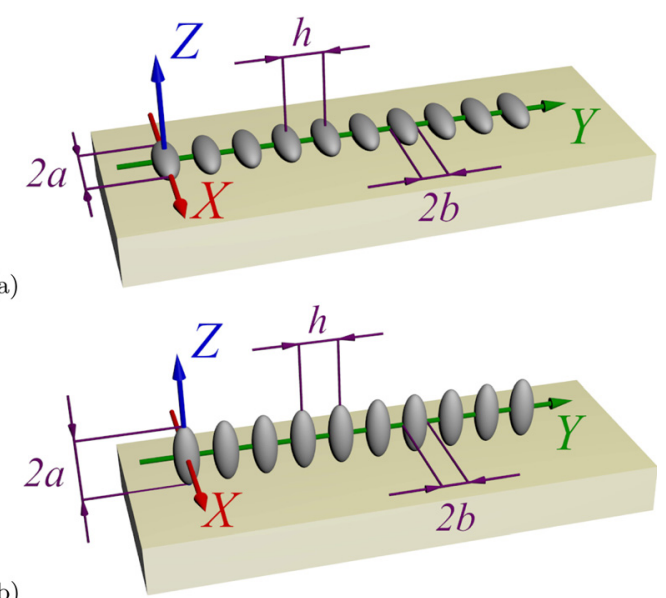

(b)

FIG. 4. Schematic view of particle chains composed of prolate spheroids whose axes of symmetry are parallel to the substrate and aligned with the $X$-axis (a) and whose axes of symmetry are orthogonal to the substrate and aligned with the $Z$ axis (b).

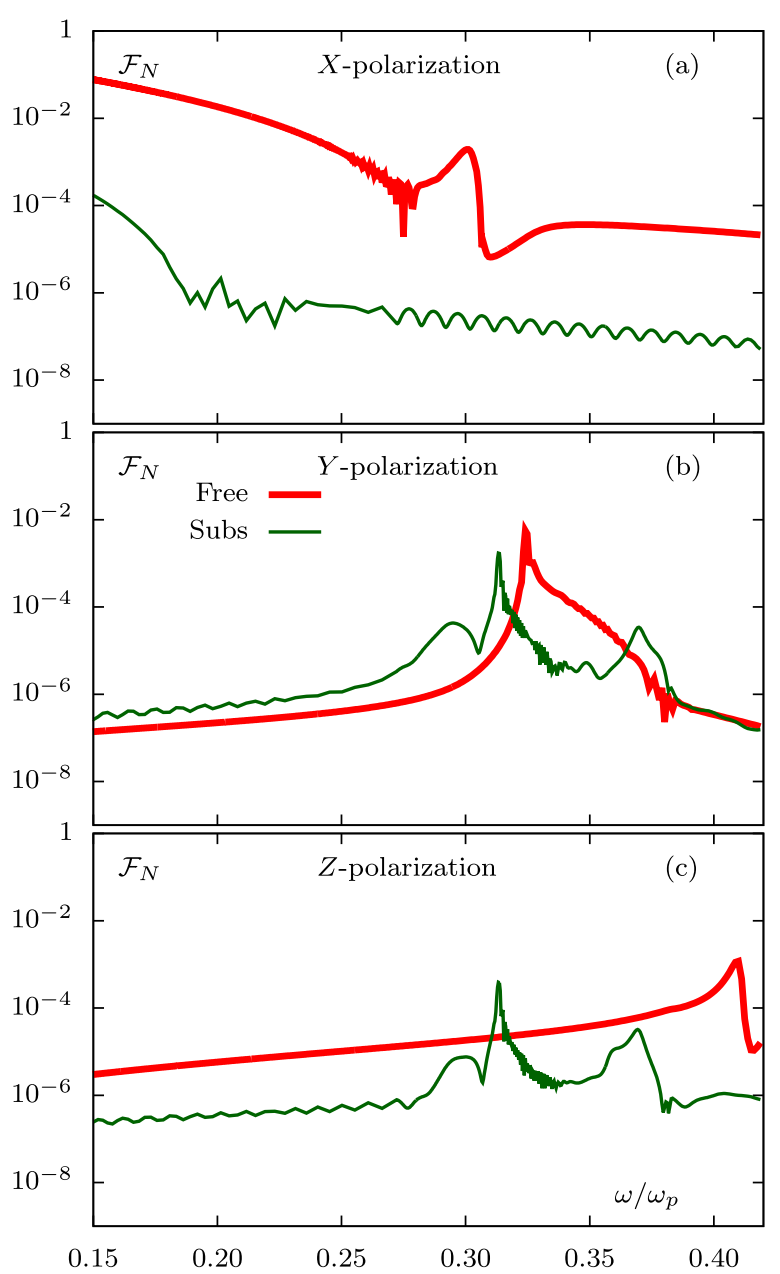

FIG. 5. Transmission spectra of chains shown in Fig. 4(a) with $N=1001$ particles. The chain either touches the substrate (thinner, green line, marked "Subs") or there is no substrate at all (thick, red line, marked "Free"). The aspect ratios of all spheroids are $\xi=0.2$. Polarization of the exciting field, A, as labeled.

explained by noting that, in the first case, the centers of spheroids are located further away from the substrate surface by the factor $1 / \xi=5$ (when both chains are touching the substrate). But this effect is not very strong. What seems to be far more important here is the following. The transmission properties of a free chain are optimal when the SPP polarization coincides with the direction of the longer axes of the spheroids. In this case, large dipole moments can be excited in the particles (compared to the case of spheres or polarization along the shorter axes), and the electromagnetic coupling in the chain is strong. On the other hand, we have seen that SPPs that are polarized orthogonally to the chain and in the plane of the substrate (such SPPs do not experience polarization conversion) are strongly suppressed by the substrate at most (but not all) frequencies. In the arrangement (a), the polarization that is aligned with the longer axes of the spheroids happens to be in the plane of the substrate. In the arrangement (b), the polarization can be simultaneously aligned with the longer axes of the spheroids and be perpendicular to the substrate. This appears to be the most advantageous arrangement for transmission. It is true that the SPPs undergo in this case polarization conversion, but the 


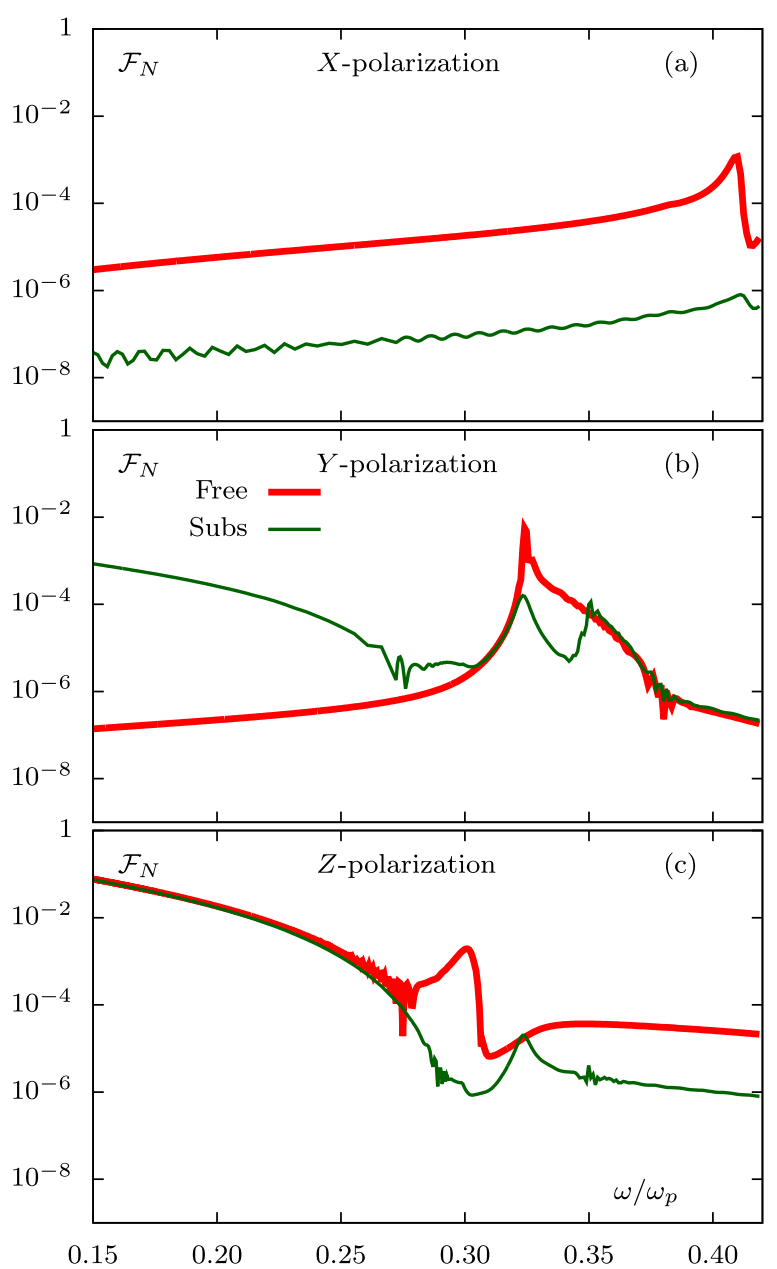

FIG. 6. Same as in Figure 5, but for a chain of prolate spheroids with axes of symmetry being parallel to $Z$ axis.

projections of the dipole moment vectors onto the $Z$ axis remain sufficiently large upon propagation; besides, polarization conversion from $Z$-direction to $Y$-direction is a weak effect at low frequencies.

We note that in $Y$ and $Z$ polarizations (which are coupled by polarization conversion) there are frequency intervals at which the substrate does not influence propagation at all. At these frequencies, the SPPs propagating in the chain are not electromagnetically coupled to the substrate with any noticeable efficiency. We will see below that this situation is typical for chains of oblate spheroids.

Finally, we note that the apparent improvement of transmission in $Y$ polarization at low frequencies (Fig. 6(b)) is due to polarization conversion. Here, the substrate does not really assist propagation in any way (as it may seem from the figure) but simply mediates the conversion of the SPP polarization from $Y$ to $Z$, which then propagates efficiently through the chain, as can be seen in Fig. 6(c). One should be careful not to attribute this improvement in transmission to the substrate: the same result could have been achieved by using $Z$ polarization from the start. The relevant effect of polarization conversion is illustrated in Fig. 7. It can be seen that the initial $Y$-polarization of the SPP is almost totally converted to $Z$-polarization. In this case, the initial and final polarizations are both linear.

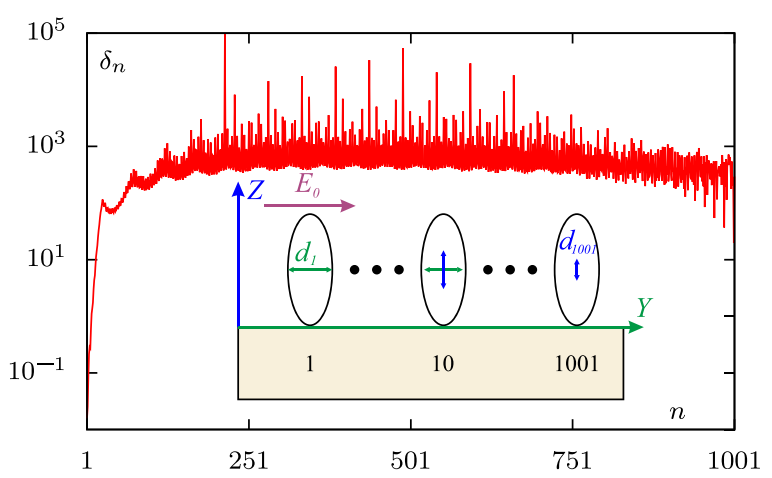

FIG. 7. The depolarization ratio $\delta_{n}(6)$ for a chain of $N=1001$ prolate spheroids oriented as in Fig. 4(b). The inset shows a schematic representation of the dipole moments transformation, which takes into account decay of the dipole amplitudes upon propagation. The $n=1$ spheroid is excited at the frequency $\omega=0.2 \omega_{p}$ with the initial polarization along the $Y$ axis.

\section{Oblate spheroids}

A schematic view of a chain of oblate spheroids on a substrate is shown in Fig. 8. The corresponding transmission spectra are shown in Fig. 9. Here, as in the case of prolate spheroids, particles have the aspect ratio $\xi=b / a=0.2$. It can be seen that, for most frequencies considered, the effect of the substrate on the transmission properties of the chain is negligible. There are some relatively narrow frequency bands in which electromagnetic coupling between the chain and the substrate is apparently strong, which causes suppression of transmission. But all these bands are at high frequencies. At the frequencies below $\sim 0.3 \omega_{p}$, which are of primary interest in waveguiding applications, transmission of a chain on a substrate is virtually indistinguishable from the transmission of a free chain. We note that the transmission is also very efficient in absolute terms-the normalized Green's function $\mathcal{F}_{N}$ can be close to unity in a very long chain consisting of $N=1001$ particles and having the physical length of $24 \mu \mathrm{m}$. This effect does not violate energy conservation and is similar to what we have observed previously in free chains. $^{21}$ Note that the transmission can, in principle, be even larger than unity without violating any conservation laws. As in the case of free chains, this effect is explained by strong coupling between the particles and the high values of the SPP group velocity. We thus conclude that the nondecaying SPPs that were described in Ref. 21 can also exist if the chain is deposited on a dielectric substrate under the condition that the right shape of spheroids is used.

The negligible role of the substrate for the chain of oblate spheroids can be explained by several factors. The most important factor is the high localization of the

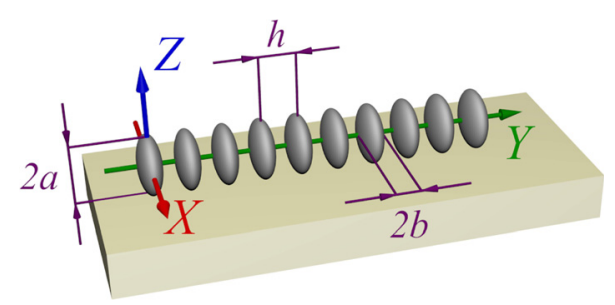

FIG. 8. Schematic view of a linear chain composed of oblate spheroids. 


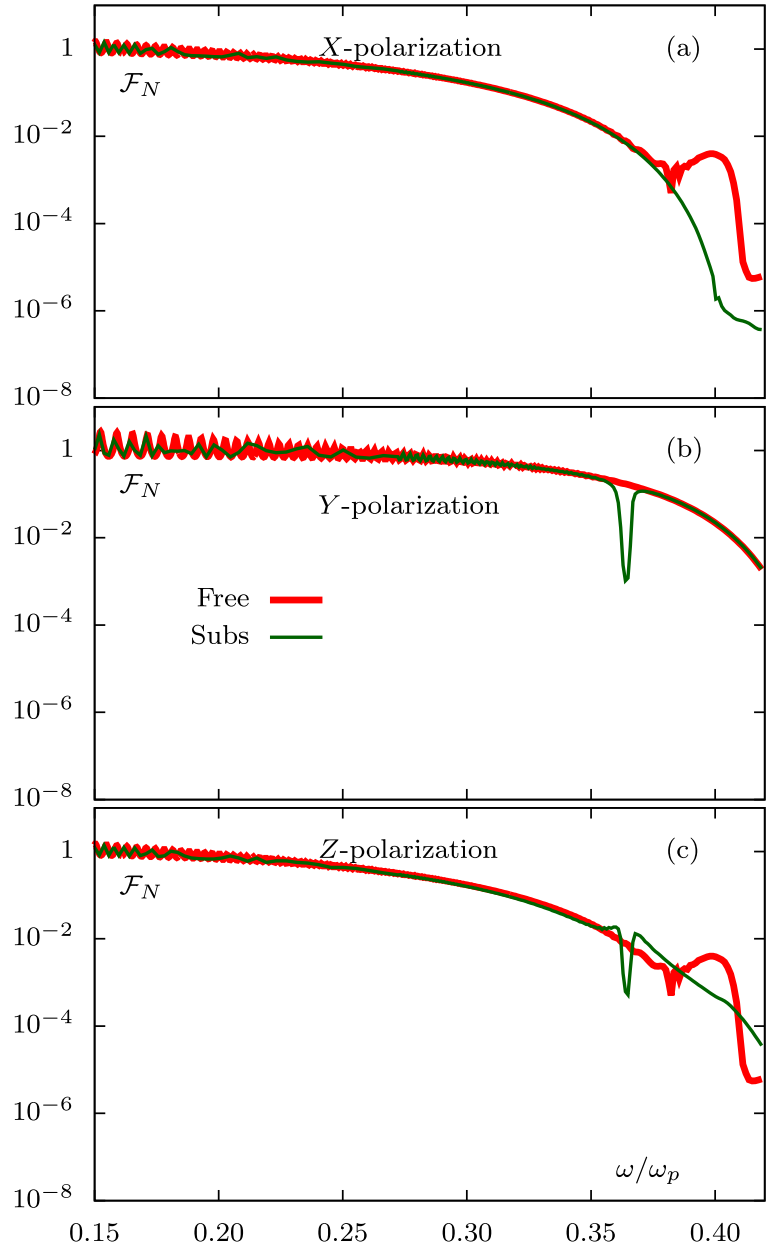

FIG. 9. The same as in Fig. 5 but for oblate spheroids. $N=1001$ and $\xi=0.2$.

electromagnetic field near the chain, which is, generally, characteristic of the oblate spheroids with sufficiently small aspect ratios. $^{22}$ Consequently, the field of the SPP does not effectively penetrate into the substrate. The second factor is the increase of particle volume relative to the cases of spheres and prolate spheroids (by the factors of 25 and 5, respectively). This results in increased particle polarizability and stronger electromagnetic coupling of the individual particles.

\section{CONCLUSIONS}

The presence of a substrate is indeed problematic for waveguiding applications of plasmonic nanoparticles chains if the particles have the shapes of spheres or prolate spheroids. There exist some combinations of parameters for which the problem is not very severe, but in general these shapes should be avoided. However, chains of oblate spheroids of sufficiently small aspect ratio experience no adverse effects of the substrate. Therefore, we conclude that the use of oblate spheroids (nanodisks) in plasmonic waveguiding applications can be advantageous when compared to other particle shapes. We note that SPPs propagate efficiently along the chain of oblate spheroids on a substrate such as the one shown in Fig. 8 in all three possible polarizations: one longitudinal and two transverse.
One of the reasons why nanodisks may be better candidate particles for waveguiding applications is that the nanodisks have a larger volume compared to other shapes such as nanoneedles (with the same values of $b$ and $\xi$ ) or nanospheres (with the same values of $b$ ). Therefore, nanodisks experience stronger electromagnetic coupling when compared to geometrically similar chains consisting of other particles. For example, the volume of the oblate spheroids we have considered is larger by the factors of 5 than the volume of prolate spheroids and by the factor 25 than the volume of spheres; yet the surface-to-surface separation of the neighboring particles is the same in all cases and the overall physical length of the chain is also the same $(24 \mu \mathrm{m})$. The other advantage is that the nanodisks tend to localize the field of a propagating SPP inside the gaps, which helps prevent parasitic couplings and losses.

The plasmonic chains considered in this paper can be fabricated by means of layer-by-layer nanolithographic technique for vertical deposition of nanodisks onto each intermediate layer with subsequent placement of a sandwich structure horizontally on a dielectric substrate. Another way of experimental realization is to use nanorods instead of prolate spheroids or thin truncated cylinders (or even parallelepipeds with a square profile) instead of oblate spheroids. What is important here is the depolarization factor for these types of particles (see the expression for polarizability tensor of nanoparticles in Ref. 22). Fabrication of chains of nanoellipsoids of rather general shape deposited on a SOI substrate (e.g., with the semiaxes of $100 \mathrm{~nm} \times 40 \mathrm{~nm} \times 15 \mathrm{~nm}$ ) has been reported in Ref. 41. In this reference, the waveguiding functionality in chains of up to $N=50$ nanoparticles has also been demonstrated. Experimental realizations of more symmetric nanodisk chains have also been reported. ${ }^{42,43}$ We therefore believe that experimental fabrication and electromagnetic characterization of the nanodisk chains described above are a realistic task, although we expect that somewhat shorter chains will be used in experiments, at least initially.

\section{ACKNOWLEDGMENTS}

This work has been carried out thanks to the support of the A*MIDEX project (No. ANR-11-IDEX-0001-02) funded by the "Investissements d'Avenir" French Government program, managed by the French National Research Agency (ANR) and was also supported in part by the U.S. National Science Foundation under Grant No. DMS1216970 and by the Ministry of Education and Science of the Russian Federation under Contract No. 1792.

${ }^{1}$ A. Nikitin, A. Kabashin, and H. Dallaporta, Opt. Express 20, 27941 (2012).

${ }^{2}$ T. V. Teperik and A. Degiron, Phys. Rev. B 86, 245425 (2012).

${ }^{3}$ T. Cheng, C. Rangan, and J. E. Sipe, J. Opt. Soc. Am. B 30, 743 (2013).

${ }^{4}$ P. J. Compaijen, V. A. Malyshev, and J. Knoester, Phys. Rev. B 87, 205437 (2013).

${ }^{5}$ A. Nikitin, T. Nguyen, and H. Dallaporta, Appl. Phys. Lett. 102, 221116 (2013).

${ }^{6}$ C. Lee, M. Tame, C. Noh, J. Lim, S. A. Maier, J. Lee, and D. Angelakis, New J. Phys. 15, 083017 (2013).

${ }^{7}$ R. S. Savelev, A. P. Slobozhanyuk, A. E. Miroshnichenko, Y. S. Kivshar, and P. A. Belov, Phys. Rev. B 89, 035435 (2014). 
${ }^{8}$ A. Vitrey, L. Aigouy, P. Prieto, J. M. Garca-Martn, and M. U. Gonzlez, Nano Lett. 14, 2079 (2014).

${ }^{9}$ L. Lin and Y. Zheng, Nanoscale 7, 12205 (2015).

${ }^{10}$ B. D. Thackray, P. A. Thomas, G. H. Auton, F. J. Rodriguez, O. P. Marshall, V. G. Kravets, and A. N. Grigorenko, Nano Lett. 15, 3519 (2015).

${ }^{11}$ V. A. Markel, J. Phys. B 38, L115 (2005).

${ }^{12}$ M. Quinten, A. Leitner, J. R. Krenn, and F. R. Ausennegg, Opt. Lett. 23, 1331 (1998).

${ }^{13}$ S. A. Maier, P. G. Kik, and H. A. Atwater, Appl. Phys. Lett. 81, 1714 (2002).

${ }^{14}$ S. A. Maier, P. G. Kik, and H. A. Atwater, Phys. Rev. B 67, 205402 (2003).

${ }^{15}$ C. Girard and R. Quidant, Opt. Express 12, 6141 (2004).

${ }^{16}$ M. L. Brongersma, J. W. Hartman, and H. A. Atwater, Phys. Rev. B 62, R16356 (2000)

${ }^{17}$ D. S. Citrin, Opt. Lett. 31, 98 (2006).

${ }^{18}$ A. B. Evlyukhin and S. I. Bozhevolnyi, Laser Phys. Lett. 3, 396 (2006).

${ }^{19}$ K. B. Crozier, E. Togan, E. Simsek, and T. Yang, Opt. Express 15, 17482 (2007).

${ }^{20}$ A. A. Govyadinov and V. A. Markel, Phys. Rev. B 78, 035403 (2008).

${ }^{21}$ I. L. Rasskazov, S. V. Karpov, and V. A. Markel, Opt. Lett. 38, 4743 (2013).

${ }^{22}$ I. L. Rasskazov, S. V. Karpov, and V. A. Markel, Phys. Rev. B 90, 075405 (2014).

${ }^{23}$ V. A. Markel and A. K. Sarychev, Phys. Rev. B 75, 085426 (2007).

${ }^{24}$ Y. Hadad and B. Z. Steinberg, Phys. Rev. B 84, 125402 (2011).

${ }^{25}$ B. Auguie and W. L. Barnes, Opt. Lett. 34, 401 (2009).
${ }^{26}$ M. S. Scheurer, M. D. Arnold, J. Setiadi, and M. J. Ford, J. Phys. Chem. C 116, 1335 (2012).

${ }^{27}$ M. Conforti and M. Guasoni, J. Opt. Soc. Am. B 27, 1576 (2010).

${ }^{28}$ S. Campione, S. Steshenko, and F. Capolino, Opt. Express 19, 18345 (2011).

${ }^{29}$ I. B. Udagedara, I. D. Rukhlenko, and M. Premaratne, Opt. Express 19, 19973 (2011).

${ }^{30}$ D. Van Orden, Y. Fainman, and V. Lomakin, Opt. Lett. 35, 2579 (2010).

${ }^{31}$ P. J. Compaijen, V. A. Malyshev, and J. Knoester, Opt. Express 23, 2280 (2015).

${ }^{32}$ D. Van Orden, Y. Fainman, and V. Lomakin, Opt. Lett. 34, 422 (2009).

${ }^{33}$ L. Novotny and B. Hecht, Principles of Nano-Optics (Cambridge University Press, New York, 2006).

${ }^{34}$ Y. N. Gartstein and V. M. Agranovich, Phys. Rev. B 76, 115329 (2007).

${ }^{35}$ I. L. Rasskazov, S. V. Karpov, and V. A. Markel, Opt. Spectrosc. 115, 666 (2013).

${ }^{36}$ A. Moroz, J. Opt. Soc. Am. B 26, 517 (2009).

${ }^{37}$ B. T. Draine, Astrophys. J. 333, 848 (1988).

${ }^{38}$ A. Sommerfeld, Ann. Phys. (Leipzig) 333, 665 (1909).

${ }^{39}$ A. A. Maradudin and D. L. Mills, Phys. Rev. B 11, 1392 (1975).

${ }^{40}$ G. Y. Panasyuk, J. C. Schotland, and V. A. Markel, J. Phys. A 42, 275203 (2009).

${ }^{41}$ M. Fevrier, P. Gogol, A. Aassime, D. Bouville, R. Megy, and B. Dagens, Appl. Phys. A 109, 967 (2012).

${ }^{42}$ R. Huber and D. Grundler, Proc. SPIE 8100, Spintronics IV 8100D (2011).

${ }^{43}$ Z. Lin, H. Xu, Y. Xu, X. Pang, Y. He, J. Jung, and H. Xia, in APS March Meeting (APS, 2015), p. W41.014. 